\title{
Actions publiques de l'Etat et développement des territoires au Cameroun : les cas du Complexe Industrialo-Portuaire de Kribi et du Barrage Réservoir de Lom Pangar autour des localités de Kribi au Sud et d'Ouami à l'Est
}

\author{
Richard Désiré EBELE ONANA \\ Chargé de cours \\ Université de Douala \\ Et \\ Merveille Brynda NGOMOU ELAT \\ Chercheure \\ Université de Yaoundé II
}

\begin{abstract}
Résumé
L'Etat du Cameroun dans son ambition de pallier aux résultats mitigés des politiques initiées par la Communauté Financière Internationale, s'est engagé à travers une nouvelle politique économique de réajuster l'ajustement structurel. Cette nouvelle politique économique a été conceptualisée dans le cadre du Document Stratégique pour la Croissance et l'Emploi (DSCE) et matérialisée dans la période 2010-2019. La mise en œuvre de l'action publique de l'Etat avec la construction des infrastructures de transport et d'énergie n'a pas permis d'atteindre les objectifs escomptés dans la période d'implémentation du DSCE sur le plan national. Toutefois, pris individuellement, certains projets ont participé au développement des territoires ou de certaines localités. Ainsi, La construction du Port en eau profonde de Kribi a contribué au développement de Kribi et ses environs à travers la transformation physique de la ville, l'urbanisation et la création des activités à caractère industriel, économique et social ; pendant que le barrage de Lom Pangar quant à lui, favorisait le développement de la pêche continentale et le développement des activités connexes, de même que des activités économiques et sociales des localités environnantes.
\end{abstract}

Mots clés : Actions publiques de l'Etat, développement des territoires, complexe industrialo-portuaire, barrage réservoir

\begin{abstract}
In its resolve to alleviate the mixed results of the implementation of policy designed by the International Financial Community, the State of Cameroon adopted a new economic policy in order to reverse the negative effects of structural adjustment. This new economic policy was encapsulated in the Strategic Document for Growth and Employment (SDGE) which was implemented from 2010 to 2019. Even though, from the assessment of the SDGE carried out in 2019, its objectives were not achieved, it appears that the realisation of some of its projects brought about some unexpected positive changes for local communities. In effect, the materialisation of the State's Public Action through the building of transportation and energy infrastructure has laid ground for the development of some territories at the local level. Then, the construction of the Deep Sea Port in Kribi has contributed to the development of the town and its surroundings by transforming its landscape, accelerating urbanisation and creating industrial, economic and social activities. In addition, the construction of the Lom Pangar dam has triggered the development of continental fishing and related activities, as well as economic and social activities in neighbouring villages.
\end{abstract}

Key Words: State Public Action, Local development, industrial port complex, reservoir dam

\section{Introduction}

Le diagnostic posé par la communauté financière internationale sur les économies africaines au sud du Sahara avait donné comme résultat, que l'Etat en Afrique était le principal responsable de sa situation de crise économique, du fait de son poids exorbitant dans l'économie nationale. La solution prônée par celle-ci pour sortir de cette situation de crise fut de mettre à mort l'Etat ou, à tout le moins, de le réduire à sa plus simple expression dans la gestion des affaires économiques (Touna Mama 2006 : 453). Pendant environ deux décennies, la communauté financière internationale, à travers l'application des Programmes d'Ajustement Structurel (PAS), a érigé sa réflexion en pensée unique, et a dicté un mode de gestion et de régulation de la vie économique aux pays africains conformément aux préceptes de Washington. Cette pensée qui faisait du marché un paradis sur terre et l'Etat un enfer (Touna Mama 2006 : 453), a consacré la mise en vacance de ce dernier.

Les résultats obtenus pendant l'application des PAS ont contribué de manière significative et profonde à l'affaiblissement de la capacité de l'Etat à produire et à permettre le bien-être de ses populations. 
La croissance économique, le développement et les équilibres souhaités par l'Etat et annoncés par les Institutions de Brettons Wood sont demeurés des vains mots. Le désengagement de l'Etat du secteur productif a conduit le pays vers une dégradation progressive des indicateurs économiques et sociaux (Fambon \&Tach 2008 :49). Conscient des performances négatives de l'économie enregistrées pendant la période d'application des Programmes d'Ajustement Structurel, mais surtout soucieux de renverser la courbe et d'atteindre son émergence à l'horizon 2035, le Cameroun grâce à sa nouvelle politique, va réajuster sa vision économique et sociale à travers la conceptualisation et la mise en œuvre du Document Stratégique pour la Croissance et l'Emploi (DSCE). Cette nouvelle politique de l'Etat a porté sur le recadrage et la projection de l'action publique économique et sociale. Pour y parvenir, l'Etat va s'attarder sur la stratégie de relance économique comme nécessité de prospection et de projection des politiques et actions publiques à travers l'importance de la mise en perspective d'une vision sur l'accès à la modernité ; à travers un maillon qui est la mise en cohérence budgétaire des actions de développement de l'Etat (Touna Mama 2008: 375). Par ailleurs, l'Etat s'est proposé de mettre en exergue le DSCE comme document de base de la stratégie de l'Etat dans les secteurs de l'économie à travers le développement des infrastructures, de la production ainsi que dans les secteurs socioculturels. Pour la relance de son économie, plusieurs secteurs clés seront mis à contribution parmi lesquels : le secteur de l'énergie et le secteur des transports.

Les investissements dans le secteur de l'énergie relevaient des domaines de la production d'énergie, de la distribution, de l'entretien, de la réhabilitation et du développement de la capacité énergétique du pays. Ils avaient pour objectif majeur de résorber à moyen terme le déficit structurel du pays en matière énergétique, d'exporter et de commercialiser sa production au-delà des frontières et porter sa capacité productrice d'énergie à environ $3000 \mathrm{MW}$ à l'horizon 2020. Parmi ces réalisations, on retrouvait la construction et la mise en eau du barrage réservoir ou entonnoir de Lom Pangar, dont l'objectif était de réguler le débit de l'eau dans le fleuve Sanaga et saturer par conséquent les centrales hydroélectriques de Song Loulou et d'Edéa (Touna Mama 2008 : 375).

Les actions envisagées dans le secteur des transports, devaient prendre en considération un ensemble de soussecteurs tels que : le sous-secteur du transport routier, le sous-secteur des transports maritimes, le sous-secteur des transports ferroviaires. Dans son ambition d'émergence, l'Etat va mettre l'accent sur l'aménagement et la construction du Port en eau profonde de Kribi.

Même si les objectifs assignés à la réalisation de ces projets n'ont pas été globalement atteints sur le plan national, et dans la période envisagée par le DSCE (Amanya 2020 : 170), il est à noter que la construction du Complexe Industrialo-Portuaire de Kribi (CIPK) et la construction du barrage réservoir de Lom Pangar se présentent comme des «fenêtres d'opportunité» de développement dans la nouvelle trajectoire de l'économie du Cameroun et spécifiquement, elles se présentent comme des instruments participant au développement des territoires ou des localités où elles sont implantées.

La construction des politiques infrastructurelles au Cameroun s'est circonscrite dans une vision du progrès possible porteuse du «vrai » changement. Les questionnements nouveaux sur les manières de conduire les politiques publiques et économiques dans les pays sous-développés ont suscité une action publique réorientée vers des modèles néolibéraux, s'inspirant de la régulation par les marchés. L'action publique ne doit plus seulement être utile, elle doit également apporter une valeur ajoutée (Sauret 2013 : 52). A travers une action publique collective (l'action se construisant au cœur des interactions sociales et non plus simplement au sommet de l'Etat) (Roux\&Savarese 2018), l'approche par l'instrumentation, nous permet de saisir autrement l'intervention de l'Etat à travers les instruments qui structurent ses programmes (Le Gales et Lascoumes 2005). Il s'agit de ne pas s'attaquer aux projets en tant qu' «unités d'analyse», mais plutôt comme «objets d'analyse ». L'instrument "projet» devient donc un mode d'action de l'autorité publique (Pinson 2005 : 200). Ayant ramené très souvent l'étude du rôle des infrastructures à l'analyse macro-économique de la croissance et du développement, la «nouvelle économie géographique » (Krugman 1991), avec le renouveau de l'analyse spatiale et régionale, justifie le retour à une analyse de base des relations entre l'infrastructure et le développement (Henner 2001 : 39f). Pour elle, les infrastructures interagissent avec les territoires des zones concernées et des zones voisines.

Revenant à la préoccupation centrale qui est celle de la relation entre action publique et développement des territoires, on se demande dès lors comment les politiques infrastructurelles, par la construction d'ouvrages de grandes envergures participent de façon efficiente et efficace au développement des territoires ou tout simplement au développement local du pays. Autrement dit, comment l'implémentation de ces deux projets, a-t-elle contribué au développement de la ville de Kribi et ses environs dans la Région du Sud et des localités de Deng Deng, Ouami, etc. dans l'arrondissement de Bélabo, Région de l'Est'

\footnotetext{
${ }^{1}$ Les éléments qui ont servi de terreau fertile à cet article se sont basés sur les recherches documentaires (livres, journaux et documents officiels). Nos données s'appuient également sur des interviews avec le personnel (ingénieurs et administratifs) du comité de pilotage et de suivi de la réalisation du complexe industrialo-portuaire de Kribi (CIPK) et du barrage de Lom Pangar. Sans négliger l'importance des observations participantes effectuées lors de nos multiples descentes aux services du port, du barrage et des terres environnantes.
} 
Le CIPK et le barrage de Lom Pangar sont des «fenêtres d'opportunité » du développement local car, ils ouvrent la voie à d'autres types d'investissements. L'émergence des dispositifs inédits de projets traduit l'insuffisance des outils existants. Le retard des pays du Sud se rattrape selon A. Gershenkron par des innovations institutionnelles permettant de subvenir aux manquements des pays en développement en termes de facteurs de production (Ngomou 2019 : 16). Ces deux grandes réalisations apportent une plus-value certaine aux territoires dans lesquels elles sont implantées. ASCHAUER note qu'il y a trois façons de modéliser la variable infrastructure dans les modèles de croissance endogène (Romer 1994). Selon lui (Aschauer 1993), l'infrastructure est un input direct dans la fonction de production ; l'infrastructure peut rendre le capital privé et le travail plus productif ; l'infrastructure est un facteur catalyseur permettant de capter des facteurs de production d'autres régions ou d'investissements directs étrangers. Dès lors ces infrastructures produisent des externalités positives qui jouent un rôle bien ciblé et capable d'agir durablement sur le développement des localités.

Cette réflexion révèle un intérêt tant empirique qu'heuristique, en ce sens qu'elle permet de ressortir les perspectives et les dynamiques évolutives des territoires à travers l'action publique de l'Etat. La construction de cette thématique invite à une interdisciplinarité entre la science politique, la géographie et l'économie. Concrètement, notre analyse se situe dans le champ de l'économie politique et de l'action publique. S'il est vrai que, la modification environnementale des espaces qui accueillent cette catégorie d'ouvrages impulse une adaptation psycho-sociale des populations à l'urbanisation et à la modernisation, il est d'autant plus vrai qu'outre la mutation socio-environnementale, le foisonnement d'activités économiques divers souscrit les «projets structurants » au modèle de l'instrumentation de l'action publique de l'Etat (Halpern et al. 2014) pour le développement du territoire national.

Le recours au néo institutionnalisme méthodologique dans sa dimension économique (Rizza 2008), nous permet de cerner les infrastructures comme des dispositifs instituant la croissance et le développement socio-économique. De même, Robert K. MERTON (Merton 1966), parlant des fonctions manifestes et latentes, traite des infrastructures en tant que projets catalyseurs de facteurs de production et porteurs de croissance.

En montrant la relégitimation de l'Etat à travers l'implémentation des politiques infrastructurelles pour l'évolution socio-économique, l'analyse évoluera dans la démonstration du Complexe industrialo-portuaire comme un hub industriel dans la localité de Kribi et ses environs(I) et du barrage réservoir de Lom Pangar comme un moteur de développement local par l'activité de la pêche dans les localités concernées (II).

\section{Le Complexe industrialo-portuaire, une opportunité de développement pour la ville de Kribi et ses environs}

Le concept de « développement» désigne l'ensemble des évolutions positives d'une zone géographique ou d'une population, que ce soit sur le plan démographique, sanitaire, culturel ou social (Ngomou 2019: 31). De façon générale, la notion de développement est conçue comme l'essor, l'expansion et même la croissance des régions et de leurs populations. Le Professeur Valentin NGA NDONGO le définit comme « un processus dynamique de changement de l'environnement, naturel ou sociétal en vue de la transformation de l'humaine condition » (Nga Ndongo1998 : 43). Il peut aussi se présenter comme « un processus de transformation des structures économiques, sociales, mentales, culturelles, institutionnelles permettant l'apparition de la croissance » (Kuate $1998: 80$ ). Dans le cadre de cette réflexion, l'on est amené à faire le constat selon lequel, le Complexe Industrialo Portuaire de Kribi contribue à induire des externalités positives dans le développement de la ville de Kribi et ses environs. Ces externalités se présentent en termes de modernisation de cette ville à travers l'émergence d'une zone industrialoportuaire qui a favorisé la transformation physique de la ville de Kribi et le développement socio-économique de ladite ville et ses environs.

\section{Le développement socio-économique de la ville de Kribi et ses environs: l'émergence d'une zone industrialo- portuaire}

Le but escompté par la construction du complexe portuaire de Kribi était que celui-ci soit le poumon économique du Cameroun à travers la création des opportunités économiques nouvelles, l'implantation des usines et des entreprises, qui pourraient soutenir l'économie nationale. Avec la construction du CIPK, la ville traditionnellement identifiée comme Cité balnéaire et touristique, vire lentement et sûrement à l'industriel. Il émerge alors de nouveaux corps de métiers qui favorisent le développement économique et social par des opportunités sur le plan commercial, réclamant une importante main d'œuvre qualifiée ou non. L'activité portuaire à Kribi a fait surgir un nouveau lexique qui se vulgarise désormais dans les discussions quotidiennes des populations.

La jeunesse se mue soit pour saisir l'opportunité d'intégrer les services du port qui regorgent d'activités nouvelles à la portée du personnage qualifié, soit pour rejoindre les entreprises en relation avec l'exploitation portuaire qui se [dé]multiplient pour de meilleures prestations de services, en ne faisant fi de l'exploitation minière.

\section{L'émergence de nouveaux corporatismes dans la ville de Kribi}


La réalisation du Port a permis la création de nouvelles activités liées à l'exploitation portuaire comme l'entreposage, l'acconage, la manutention, le remorquage, le lamanage, la consignation, le stockage, la gestion des terminaux, le pilotage, le transit, le ravitaillement des navires, le fret, etc. Ces activités sont dirigées par la Kribi Container Terminal (KCT) ; avec près de 300 collaborateurs répartis dans différents corps de métiers opérationnels à ce jour. Son engagement socio-économique affiche près de 400 emplois directs et $+/-150$ emplois indirects dont $90 \%$ des collaborateurs sont de l'Océan ${ }^{2}$. En plus, $70 \%$ des employés ont moins de 35 ans avec $10 \%$ de femmes dans les métiers opérationnels. Les projections du KCT font état de 22 milliards de FCFA de revenus générés par an et de 300 emplois directs. Le directeur général du PAK, Patrice MELOM, va plus loin et envisage à peu près 2000 emplois directs et 2000 emplois indirects ${ }^{3}$. L'exploitation du terminal à conteneurs a été concédée à Bolloré Africa Logistics, CMA-CGM et la China Harbour Engineering Company (CHEC), qui se sont tous les trois regroupés au sein de la société holding appelée KTH (Kribi holding terminal) ${ }^{4}$ et à certains actionnaires camerounais ${ }^{5}$.

Le terminal polyvalent qui devra accueillir les marchandises non-conteneurisées, a une capacité nominale d'hébergement de 1,2 millions de tonnes de produits par an. La gestion de ce dernier a été concédée au groupement constitué par le logisticien français Necotrans et KPMO (Kribi Port Multi-Operators) actuellement sous-traitant du terminal.

Compte tenu de la complexité des activités, la société chargée du développement de la technopole (la STDKO) sera structurée en une holding avec huit branches, spécialisées chacune dans une activité (port, aéroport, pêche, agriculture, etc.). A la clé, 1 million d'emplois directs devraient en résulter ${ }^{6}$.

La réalisation du port a permis également la création de plusieurs usines de transformation et d'exploitation du bois. Ainsi, on retrouve des entreprises qui font dans l'exploitation du parc à bois et aires de stockage du CIPK, notamment Kribi Development Company Sarl (KRIDEVCO), Société d'Exploitation des Parc à Bois du Cameroun (SEPBC), Agence de Prestation Maritimes S.A (APM), Etablissements Sali Ndjida, Bolloré Transport Logistics (BTL), etc ${ }^{7}$. En réalité, avant la construction du complexe industrialo-portuaire, Kribi n'était port que par rapport à l'embarquement du bois. Ainsi l'ouverture du parc à bois de Kribi a pallié l'une des causes d'engorgement du port de la capitale économique ${ }^{8}$. En dépit de la situation d'amélioration au port de Douala, «les expéditeurs de sciages et de grumes en République centrafricaine ont détourné leurs exportations vers le port de Kribi ${ }^{9}$. Ceci pourrait donc être un atout particulier pour les exportateurs et investisseurs de la transformation du bois qui contribue déjà à hauteur de $4 \%^{10}$ au PIB après le pétrole, le café et le cacao.

Avec l'installation de ce parc, des emplois ont été générés à travers le recrutement d'une main d'œuvre locale qualifiée ou non. Ces emplois, à durée déterminée ou à durée indéterminée, ont concerné préférentiellement les jeunes du département de l'Océan et ceux des villages riverains au site du projet.

$\mathrm{Au}$ rang des entreprises de transformation de certaines matières premières, l'on retrouve Biocam, un exportateur de déchets de bois servant à la production de l'électricité (biomasse). Aussi, avons-nous l'unité de transformation Atlantic Cocoa Corporation (ACC), qui a signé le 24 janvier 2018 avec le PAK, le tout premier contrat d'occupation de la zone industrielle du port en eau profonde de Kribi. Ce contrat autorise Atlantic Cocoa, filiale d'Atlantic Group, contrôlée par le milliardaire ivoirien Koné Dossongui, à construire dans la zone industrielle du

\footnotetext{
${ }^{2}$ Ces informations ont été données au cours du séminaire sur la vulgarisation des procédures au Port de Kribi, organisé par le PAK et les Douanes Camerounaises, en février 2018. Document PDF en ligne, http://www.pak.cm/wpcontent/uploads/PRESENTATION-KCT-SEMINAIRE-PAK-FEV-2018.pdf, consulté le 18 Mars 2019.

${ }^{3}$ Dans une interview publiée dans le magazine du ministère des transports du Cameroun, le directeur général du PAK annonce qu'ils sont déjà à plus de 1500 emplois créés en janvier 2020.

${ }^{4}$ Document de travail de Thierry Pairault sur Kribi : Bolloré, CMA-CGM \& CHEC[En ligne], mars 2019.

${ }^{5} \mathrm{D}$ 'après certains articles journalistiques, ces onze actionnaires camerounais ont versé leur contribution au capital social de KCT à hauteur de 20,75 milliards Fcfa, soit, 20\% des parts de l'entreprise créée pour exploiter le terminal à conteneurs du port de Kribi.

${ }^{6}$ Informations d'après l'article dans le magazine les Afriques, Algérie, numéro 128, juillet 2010.

${ }^{7}$ Répertoire des entreprises installées dans la zone industrialo-portuaire à Kribi, disponible aux services administratifs du Port Autonome de Kribi.

${ }^{8}$ En allant fermer le parc, tout le bois de la zone CEMAC a convergé au parc de Douala qui était devenu complètement insuffisant.

${ }^{9}$ Certains exploitants forestiers préfèrent désormais la plateforme de Kribi pour leurs exportations de bois. Le port de Kribi est ainsi rapidement devenu, le deuxième principal point d'évacuation du bois camerounais et Sous-Régional vers l'international, avec près de 1700 mètres-cubes de bois manutentionnés par jour selon les estimations du Port Autonome de Kribi en 2018.

${ }^{10}$ Pourcentage donné par le Centre de recherche forestière international (Cofor) lors de l'atelier sur le thème : «Gestion locale des ressources forestières et fauniques : bilan et perspectives » qui s'est tenu du 16 au 17 janvier 2019 à Yaoundé. 
port en eau profonde de Kribi, une unité de transformation de fèves de cacao d'une capacité de 48000 tonnes par an, extensible à 64000 tonnes. Le coût de cette usine est chiffré à environ 30 milliards de francs $\mathrm{Cfa}^{11}$.

Grâce au port, les opérateurs économiques auront la possibilité d'accroitre l'exportation des produits agricoles vers le marché international. Avec sa stratégie de développement agricole ${ }^{12}$, le Cameroun pourrait accroitre sa productivité agricole de $50 \%{ }^{13}$. In fine, au regard de ce qui précède, à travers ces entreprises et les multiples emplois créés, le port a permis le développement économique local qui s'est étendu au niveau national et même sous régional.

Le Cameroun dispose de considérables réserves de pétrole. Avec la construction du Complexe Industrialo-portuaire de Kribi et la réduction des impôts, l'oléoduc Tchad-Cameroun a déjà commencé à transporter le pétrole extrait des gisements situés au sud du Tchad jusqu'au port de Kribi. Le projet, soutenu par la Banque Mondiale, est porté par un consortium composé par les deux entreprises nord-américaines, Exxon-Mobil et Chevron, et la société Malaisienne Petronas.

Par ailleurs, la Centrale à Gaz de Kribi, d'une capacité de 216 MW, actuellement en cours d'extension à 330 MW est l'une des plus importantes du Cameroun. Cette infrastructure énergétique permet de valoriser le potentiel du champ Sanaga Sud. Ce potentiel a suscité un projet de construction d'une unité de liquéfaction du gaz dans la ville de Kribi, pour anticiper la production de gaz naturel liquéfié (GNL) au Cameroun dès 2017. Il est conduit par la société française Perenco, leader de la production pétrolière dans le pays, la $\mathrm{SNH}$ et son partenaire la société française Engie, anciennement connue sous l'appellation de GDF-Suez. Avec une capacité de production annuelle de 3,5 millions de tonnes de gaz, elle approvisionne déjà les marchés à l'export tout en satisfaisant la demande intérieure ${ }^{14}$.

\section{Des opportunités économiques sur le plan commercial et la création d'emplois}

Les investissements en infrastructures de développement ont des effets économiques réels et importants. Ils sont destinés à favoriser la croissance économique (Henner 2001 : 51). Le CIPK ne déroge pas à cette règle du développement; car il présente des opportunités à travers les activités logistiques et de transport, mais aussi ils incitent à des Investissements Directs Etrangers (IDE) par l'installation des grandes entreprises à caractère industriel.

Nouveau fleuron de l'économie camerounaise, le CIPK, de par sa position stratégique dans le Golfe de Guinée, est devenu un hub de logistique et de commerce dans la Région. Il assure non seulement les trafics domestiques, mais aussi les trafics dans certains pays de la Sous-région. En effet, le cadre institutionnel des ports camerounais, qui consacre l'autonomie des ports et la création des autorités portuaires, offre des possibilités de valorisation d'actifs supérieurs aux conditions d'antan, compte tenu de la forte attractivité du golfe de Guinée, riche en pétrole et en matières premières. A travers la construction du complexe portuaire de Kribi, «nombre d'analystes économiques voient déjà le cour de l'économie camerounaise battre un peu plus à Kribi qu'à Douala, l'actuelle capitale économique du pays $»^{15}$. Et les ambitions du gouvernement camerounais pour cette ville, portent à croire qu' "au cours des prochaines années, à défaut de devenir le principal pôle économique du Cameroun, Kribi sera l'un des maillons essentiels de l'économie nationale » ${ }^{16}$. L'infrastructure qui s'inscrit dans l'agenda de l'émergence du Cameroun à l'horizon 2035 va entraîner une compétitivité, créer des emplois, réduire la pauvreté et booster l'économie locale. La ville de Kribi va considérablement se métamorphoser pour devenir une ville industrielle ; avec comme effets induits l'installation de nouvelles entreprises qui vont absorber une main-d'œuvre locale à recruter sur place.

Kribi accueille des entreprises à caractère industriel telle que la société indienne Azad Coach et les Chinoises GacGonow et Yutong. Le consortium indo-chinois, qui a signé le 11 juin 2015 avec la Cameroon Automobile Industry Company (CAIC), annonce des investissements d'une enveloppe globale d'environ 92 milliards de francs CFA dans des projets de construction de deux unités ${ }^{17}$ de montage de véhicules (camions et berlines). Environ 4620

${ }^{11}$ Reportage dans le journal Cameroon tribune du 20 septembre 2018.

${ }^{12}$ La stratégie de développement agricole promue depuis 2012 par le Cameroun privilégie un modèle d'agriculture utilisant de grandes surfaces agricoles pour des monocultures destinées à l'exportation, principalement l'hévéa, le palmier à huile etc.

${ }^{13}$ L'agriculture contribue en 2019 à hauteur de $22.9 \%$ au PIB du Cameroun et engage plus de $62 \%$ de la population active. www.mediaterre.org/actu,20190927141926,6.html, consulté en mars 2020.

${ }^{14}$ Perenco Cameroun va installer une unité flottante de liquéfaction du gaz naturel au large de Kribi, mis en ligne le 03 octobre 2014, consulté en juin 2019.

${ }^{15}$ Ces données sont tirées de l'article du journaliste Brice Mbodiam publié dans le magazine économique investir au Cameroun, numéro 42 du mois d'Octobre 2015, page 8. C'est un magazine qui publie mensuellement sur l'actualité camerounaise et s'intéresse à l'économie, l'investissement, la gestion publique, les télécoms et l'agriculture.

${ }^{16}$ Ibid.

${ }^{17}$ La première sera à Kribi et l'autre à Douala. 
emplois directs devaient être générés dans le cadre de ces projets, au cours des 15 premières années d'activités. Le gouvernement se charge de mettre à disposition 900 hectares de terre dans les environs du PAK ${ }^{18}$.

Par ailleurs, dès le mois de mars 2020, Tractafric Equipement achève les travaux de construction de son unité d'assemblage des engins et groupes électrogènes dans la zone industrialo-portuaire de Kribi, dans la région du Sud du Cameroun. Ces machines seront disponibles sur le marché de la CEMAC au courant du $2^{\mathrm{e}}$ trimestre $2020^{19}$. Elle prévoit devenir la toute première usine d'assemblage des engins en Afrique centrale; avec une capacité de production de 250 machines chaque année.

La Société Industrielle de Construction d'Appareils Electroménagers et de Climatiseurs (SICAMEC) est dynamisée par Alkis Bruno Domyou Noubi, sur une superficie de 20 hectares sur ce site situé à quelques encablures de l'autoroute Kribi - Lolabe. En plus d'être la toute première entreprise à capitaux camerounais à obtenir un accord d'établissement dans la zone industrielle du port de Kribi, elle est aussi un projet porteur d'impact réel en matière de création d'emplois (1000 emplois directs), avec sa capacité de production de 15000 unités par mois (5000 climatiseurs, 5000 réfrigérateurs et 5000 congélateurs) ${ }^{20}$.

La réalisation du Complexe Industrialo-Portuaire de Kribi a par ailleurs, favorisé des opportunités commerciales importantes dans la ville de Kribi en ce sens que, ces nouvelles activités qui ont attiré plusieurs milliers de personnes en dehors des employés du port et les pêcheurs, notamment les opérateurs économiques, les commerçants, les débrouillards, ainsi que de nombreuses familles. On assiste à une vague de création de Petites et Moyennes Entreprises $^{21}$. La population, désormais hétérogène, a ainsi augmenté et celle-ci s'est lancée dans le petit commerce (buvettes, snack bar, restaurant, etc.). Se développe donc le secteur informel. Ces activités se présentent comme des opportunités de croissance, car elles permettent aux populations de vivre, de payer les impôts et taxes ; toute chose qui permet à l'économie de tourner et financer les projets locaux de développement.

L'aménagement du port de Kribi a favorisé en plus de l'augmentation de la fréquentation touristique dans la ville de Kribi, les investissements privés dans le secteur de l'hôtellerie et de la restauration, etc. Il a été noté lors de nos échanges avec diverses personnes et personnalités de la localité, qu'au débarcadère par exemple, le travail des restaurateurs (braiseuses, barmans) s'est multiplié par trois grâce à l'augmentation de la clientèle ; donnant de plus grands bénéfices malgré la rareté du poisson ${ }^{22}$. Le développement des activités économiques et le recrutement des populations riveraines ont augmenté substantiellement leurs revenus. De plus, les revenus de l'exploitation du port vont permettre, en cas de gestion responsable, l'amélioration du cadre et des conditions de vie des populations à travers la mise en œuvre des microprojets de développement dans la ville de Kribi.

\section{La transformation physique et l'urbanisation de la ville de Kribi et ses environs}

Le complexe industrialo portuaire est une infrastructure qui se rapporte à la théorie de la croissance endogène évoquée par l'économiste Paul Romer, qui démontre comment des facteurs endogènes (internes) peuvent favoriser la croissance. Elle explique le progrès économique par la technologie et approfondit l'analyse de ce progrès par les politiques que le gouvernement peut mener en matière de recherche et développement ainsi que les aides et subventions apportées par l'État pour le développement technologique et humain. Elle complète la théorie de la croissance exogène, développée par Robert Solow (Solow 1994). Selon les théories de ce modèle, la croissance économique est considérée comme un processus auto-entretenu par l'accumulation de quatre facteurs : le capital humain, le capital physique, le capital technique et le capital public. Ces variables évoluent suivant un taux d'accumulation fortement lié aux choix économiques. A Kribi, l'accumulation de ces facteurs donne lieu à une transformation physique de la ville et par ricochet à une amélioration du cadre de vie des populations.

\section{La transformation physique de la ville de Kribi}

De prime abord, la transformation physique de la ville de Kribi et ses environs, signifie une [re]modélisation du cadre de vie et une amélioration esthétique de l'apparence de la ville.

Les outputs du CIPK ont engendré un aggiornamento des populations de cette localité. En effet, les habitants de la ville de Kribi doivent s'adapter à la nouvelle infrastructure qui vient moderniser leur milieu d'existence et leur milieu de vie. Cette modification se traduit par l'aménagement et l'urbanisation de la ville, et le développement de la transaction foncière.

\footnotetext{
${ }^{18}$ Op. cit., p. 13.

${ }^{19}$ Article du journaliste Brice Mbodiam dans investir au Cameroun [En ligne], février 2020.

${ }^{20}$ Cet investissement est officiellement estimé à 45 milliards de FCFA d'après l'article du journaliste Brice Mbodiam dans investir au Cameroun, mis en ligne en Août 2019.

${ }^{21}$ Données issues de l'entretien avec le délégué départemental de l'Océan du ministère des petites et moyennes entreprises.

${ }^{22}$ Enquête de terrain menée au débarcadère près de la plage Ngoyé à l'entrée de la ville, Mai 2019. 56
} 
Parlant des projets de ville, Gilles Pinson parle de l'aménagement et de l'urbanisation comme des formes de «valorisation des atouts, des avantages comparatifs de la ville, tout ce qui peut favoriser l'implantation des entreprises et de leurs cadres » (Pinson 2008 : 204). La construction du port de Kribi a permis la construction de nouvelles infrastructures de transport, éléments indispensables pour l'expansion de la ville et ses environs. Le rôle des pouvoirs publics est d'adapter l'offre d'infrastructures à la densification spatiale des individus et des entreprises (Ngomou 2019 : 47). La Communauté Urbaine de Kribi, en réaction à cette conjoncture s'attèle à mettre en œuvre un Plan Directeur d'Urbanisme depuis 2012, qui vise à donner un nouveau visage à ce nouveau pôle de développement en gestation ${ }^{23}$. Ces infrastructures sont indispensables à l'activité économique, à la mobilité des personnes et des biens et à la transformation de la ville. En effet, la construction du Port Autonome de Kribi a permis la réalisation et le bitumage de l'autoroute Kribi-Mboro, en allant vers le site du port. La réalisation de cette autoroute a été confiée à l'entreprise China Eximbank Corporation (CHEC). Cet axe sert de trafic entre le port et le centre-ville de Kribi. Cette autoroute assurera également le trafic entre la vieille ville de Kribi et la nouvelle métropole projetée entre Grand Batanga I, Mboro et Lolabé (Boupda 2014 : 28). A moyen terme, la construction du Port de Kribi a nécessité et a permis la réorganisation et l'aménagement de l'espace urbain avec une restructuration de voirie de cette ville.

Cet aménagement concerne plusieurs infrastructures routières en cours de réalisation dans la voirie urbaine, notamment la construction des tronçons : Carrefour Tradex- Carrefour MRS- Carrefour Poste, Carrefour pont sur la Kienké-Carrefour Mokolo, Carrefour Cathédrale - Carrefour COTCO - Carrefour hôtel Tara plage-vers le CIPK, Carrefour Kingue - Stade de football -Afan Mabe - hôtel Cocotier -intersection avec la National 7, la Pénétrante Edéa - Kribi à partir de Tradex (distance $3 \mathrm{~km}$ ) (Boupda 2014 : 28). Dans cette zone, il s'agit de renforcer la route Nationale $\mathrm{N}^{\circ} 7$ de sorte qu'elle puisse répondre à un trafic de classe T3 (1000 - 3000 véhicules par jour). Cet axe fait partie de la route littorale qui dessert le Port, elle est réalisée par l'entreprise RAZEL pour la partie urbaine.

En ce qui concerne l'axe Kribi-Edéa-Campo, le tronçon long de $38,5 \mathrm{~km}$ va faciliter le transport des conteneurs qui sont transbordés sur Douala par mer. Toutes ces infrastructures ont une plus-value dans le développement local de la ville.

La transformation physique de la ville de Kribi s'est également caractérisée par une urbanisation poussée. Le Port en eau profonde a permis la montée fulgurante des transactions foncières. Le nombre d'immatriculation foncière a presque doublé d'année en année depuis 2010, atteignant plus de 20000 Titres Fonciers en $2019^{24}$. Ainsi l'on a constaté un développement exponentiel dans la ville d'un nouveau métier: celui des agents de la transaction immobilière. Ce développement a permis de créer des emplois, malgré son caractère informel, pour les jeunes de la localité et a par conséquent diminuer le taux de chômage.

Les agents immobiliers ou « intermédiaires » ou « démarcheurs », dont la fonction est de mettre en relation l'offre et la demande en matière foncière, se recrutent parmi les sans-emplois, mais aussi et surtout, parmi les agents des services municipaux, départementaux, régionaux ou centraux. Leur rôle est de faciliter l'obtention de titres de propriété aux autochtones et de chercher des acquéreurs ou bien de repérer des terrains en vente, d'en négocier les prix et parfois de se substituer au vendeur. Dans ce cas, ils réalisent un double bénéfice, en vendant d'abord le terrain plus cher que le prix demandé par le propriétaire, et en touchant ensuite leur commission sur le prix d'achat en tant qu'intermédiaires (Kuete et Assongmo 2010). Cette fonction de démarcheur s'est accrue considérablement à Kribi. De Kribi jusqu'à Grand Batanga, la pression foncière est extrêmement importante, les résidences privées et les hôtels de différents standings et de différents types, se multiplient. La demande se fait régulière et se révèle être une importante source de revenus.

\section{L'amélioration du cadre de vie des populations}

Les effets induits du CIPK à moyen terme sont perceptibles à travers la création des services infrastructurels, c'està-dire l'implémentation des mesures sanitaires, éducatives, salariales et de paix dans la recherche du développement. Ayant déjà abordé la question salariale avec les emplois créés plus haut, nous insisterons sur les services infrastructurels, qui se caractérisent par le soutien qu'ils apportent à toute activité économique pour satisfaire les besoins fondamentaux de la population (Poirot 2001 : 26f). Ici, l'Etat a porté un intérêt à la sauvegarde du bien commun par une responsabilité sociale volontaire en créant des services de santé et les services infrastructurels sociaux.

En effet, sur le plan sanitaire, la ville de Kribi ne disposait que d'une structure de service de santé de base pour les populations : l'Hôpital de District. Il était à la dimension de la ville et de sa population. Aujourd'hui, la ville se remplit et s'agrandit, et le développement de la structure était devenu inévitable. Des réformes et des améliorations

\footnotetext{
${ }^{23}$ Communauté urbaine de Kribi, Plan directeur d'urbanisme de la ville de Kribi. Maritimes en

Mutation, 2012.

${ }^{24}$ Entretien avec les délégués départementaux et le conservateur foncier du ministère du domaine du cadastre et des affaires foncières de l'Océan
} 
ont été faites sur le plan structurel et le service y est même meilleur avec les prestations des nouveaux médecins. «Le besoin en gynécologie était pressant à Kribi, car il n'y avait pas de permanent dans toute la ville. Les prestations devenaient assez coûteuses et pas toujours accessibles à tous » déclarait un personnel de santé ${ }^{25}$.

Pour pallier l'insuffisance infrastructurelle des services de santé, on assiste à un afflux d'investisseurs qui participent à la création des structures de santé, notamment la Clinique de l'Océan. A côté de ce centre, une dizaine de centres médicaux ont été créés pendant la décennie 2010-2020. C'est le cas du Centre Médical Mpumanwet Kribi, de la Clinique du Bon Secours, de la Clinique Médico-dentaire JADE, etc. Dans la même veine, l'ONG Insolàfrica (Infermeria Solidària per l'Africa), qui est une formation professionnelle médicale, offre des services dans le domaine des maladies chroniques, dans la consultation, la promotion des soins infirmiers ${ }^{26}$. Cette organisation a créé une école de soins infirmiers, pour la formation professionnelle de 120 élèves dans la ville de Kribi.

S'agissant des infrastructures éducatives, plusieurs écoles ont été construites, avec de nouvelles filières créées, notamment le Centre d'Instruction Maritime et Portuaire (CIMP) qui a ouvert en 2009, en prévision de l'ouverture du port en eau profonde. Au programme, tout un panel de formations, allant de six à sept mois avec mise en pratique et stages. Nous avons par exemple la construction de l'annexe de la structure de formation scolaire catholique Père Monti, qui a effectué sa première rentrée scolaire en septembre 2018. C'est un complexe qui dispose d'infrastructures modernes nécessaires à l'apprentissage de ses pensionnaires.

Pour ce qui est de la construction des logements, la construction du port de Kribi a engendré la relocalisation des populations autochtones. Cette relocalisation a été possible grâce à la construction des logements et à l'attribution des lots de terrains aux groupes expropriés même si les titres fonciers de ces lots ont été établis sous la responsabilité de l'Etat. Cette mesure avait pour but d'éviter que les populations ne revendent ces terrains. «Donnez-leur un terrain titré, ils iront le revendre et avec cet argent, vous les verrez mener la belle vie c'est-àdire : femmes, vin, fête... dans les hôtels huppés de la ville pendant 03jours. Et la suite? C'est le vagabondage et la misère », justifiait le $1^{\text {er }}$ adjoint préfectoral de Kribi. Au fait, les populations, dans ce mode de gestion domaniale, sont protégées d'une certaine manière ; dans la mesure où leurs biens seront sécurisés et les éviteraient de plonger dans la dilapidation ${ }^{27}$.

L'autre aspect positif en faveur de l'amélioration du cadre de vie des populations, est l'aide matérielle qui leur est apportée: les forages aquatiques, des dons en matériel médical et en matériel de travaux agricoles, ainsi que l'aménagement d'un débarcadère pour les pêcheurs. Il y a également eu la construction des forages et des puits d'eau potable dans les villages Eboundja, Nlende-Dibé, Boussibilka, Mboro et Lolabe aux alentours de Kribi (Etude d'Impact Environnemental et Social Sommaire du projet d'aménagement et d'électrification de la plage de Ngoyekribi à des fins touristiques, Yaoundé 2014).

\section{Le développement de la pêche continentale artisanale et l'amélioration des conditions de vie des populations autour de Lom Pangar}

L'énergie est importante pour tous les secteurs de l'économie. Elle fournit l'électricité nécessaire pour les activités industrielles, commerciales, des ménages et agricoles, ainsi que pour les services sociaux essentiels tels que l'éducation et la santé. Le Cameroun fait partie des pays du continent africain qui possèdent un potentiel énergétique varié et abondant (Tchinang 2011 :61). Le barrage réservoir de Lom Pangar avait pour mission principale de réguler l'eau au niveau du fleuve Sanaga et de saturer l'eau pendant la période d'étiages dans les Centrales Hydroélectriques de Song Lou Lou et d'Edéa, avec pour incidence la baisse des délestages particulièrement dans la zone d'implantation du projet et de manière générale au niveau national. Au-delà de ces deux objectifs qui semblent avoir été atteints, la construction du barrage de Lom Pangar a induit des implications économiques et sociales qui œuvrent dans le développement des localités de Belabo, Deng Deng, d'Ouami, etc. Les externalités de ce «projet structurant » s'expriment en termes de développement de la pêche artisanale continentale et de la modification du cadre de vie des populations les services infrastructurels.

\section{Le développement de la pêche artisanale continentale}

La mise en eau du barrage réservoir de Lom Pangar a permis les remontées de l'eau sur une superficie d'environ 2000 hectares. Cette montée de l'eau a attiré dans la zone de construction du barrage près de 6000 pêcheurs de nationalité diverses pour l'activité de la pêche. L'arrivée massive de ces pêcheurs venus des quatre coins du

\footnotetext{
${ }^{25}$ Enquête menée à l'Hôpital de District de Kribi en mai 2019.

${ }^{26}$ Infermeria solidària per l'Africa est une ONG qui travaille à la formation des professionnels de santé, en particulier les infirmières et l'amélioration des établissements de Santé au Cameroun. Le projet de création d'une école d'infirmière à Kribi vise à renforcer le système de santé dans la région sanitaire du Cameroun Océan. Il est mis en œuvre dans la ville de Kribi depuis Janvier 2012.

${ }^{27}$ Entretien avec Derlin Franck EYONO EBANGA, $1^{\text {er }}$ adjoint au préfet de Kribi, réalisé le 14 mars 2019. 58
} 
Cameroun et d'ailleurs, a donné à diverses localités parmi lesquelles les villages autour d'Ouami, l'allure de villes bruyantes et animées. Grâce au développement de diverses activités génératrices de revenus, les conditions de vie des populations sont moins précaires et se modernisent. Celles-ci doivent désormais s'y adapter.

\section{Développement d'Activités Génératrices de Revenus (AGR) : la pêche, le fumage et le séchage de poissons}

La retenue de l'eau à Lom Pangar a favorablement influencé les villages aux alentours, car elle a permis la concentration de nombreuses variétés de poissons dans l'eau dans le village d'Ouami. Les populations se sont donc appropriées cette opportunité et ont fait de la pêche, leur principale activité lucrative. D'autres activités connexes se sont développées autour de la pêche, notamment le fumage et le séchage de poissons.

Le succès de la pêche dans la retenue de Lom Pangar repose sur plusieurs atouts:

Une retenue d'une surface de $540 \mathrm{~km}^{2}$ et d'une capacité de 6 milliards de $\mathrm{m}^{3}$ d'eau riche de plusieurs espèces de poissons $^{28}$. Les dernières statistiques de pêche en $\mathrm{kg}$ du MINEPIA pour l'année 2017 (Rapport sur l'état de la pêche au Cameroun 2017) le témoignent dans le tableau ci-après:

\begin{tabular}{|l|l|l|l|l|}
\hline $\begin{array}{l}\text { Espèces de } \\
\text { poisson }\end{array}$ & Fumés & Frais & Séchés & Total \\
\hline Silures & 346000 & 200000 & 12000 & 558000 \\
\hline Tilapia & 260000 & 150000 & 18000 & 428000 \\
\hline Carpes et autres & 6000 & 4000 & 1000 & 11000 \\
\hline
\end{tabular}

\section{Source : Statistiques de pêche du MINEPIA en $\mathrm{kg}$}

- $\quad$ plus de 6000 pêcheurs expérimentés ;

- $\quad$ près de 7 sites de débarquement de produits de pêche (Ouami, Kokbédi, Gagouba, Touraké, Mararaba, ...);

- $\quad$ constructions de deux (02) débarcadères (Ouami et Touraké);

- $\quad$ zones favorables à l'agriculture dans la retenue après le retrait de l'eau;

- $\quad$ électrification de la zone (fonctionnement des réfrigérateurs).

A côté du développement de l'activité de la pêche se sont greffées d'autres activités connexes telles que le fumage et le séchage de poissons. L'activité de fumage qui prospère autour du quai d'Ouami, mobilise un nombre important de personnes notamment des femmes. Le séchage des poissons malgré son caractère archaïque et artisanal mobilise également une importante main d'œuvre. Ces poissons sont acheminés vers les marchés locaux et même vers les autres villes du pays. L'activité de la pêche autant que celle du fumage ou du séchage a permis la création des milliers d'emplois. Ces activités qui demeurent au stade artisanal nécessitent l'implication de l'administration publique afin de les réguler et de les réglementer. Ces activités dont l'importance n'est plus à démontrer doivent entrer dans la chaine des valeurs du pays et générer des revenus en termes de taxes et d'impôts pour la commune de Bélabo à l'Est, pour la Région de l'Est et pour l'Etat du Cameroun.

Ces activités qui se présentent comme des opportunités de croissance et de développement ont attiré plusieurs milliers de personnes, en dehors des pêcheurs notamment les opérateurs économiques, les commerçants, les débrouillards, ainsi que de nombreuses familles ${ }^{29}$. Il s'est développé à côté de ces activités qui génèrent des emplois directs, d'autres activités telles que des petits commerces, des débits de boisson autour des sites. Le barrage réservoir de Lom Pangar qui a vu se développer un pool économique autour des activités de la pêche a favorisé le développement du transport des personnes et des biens et la réduction du chômage dans ces zones concernées, la réduction de la pauvreté des populations.

\section{Encadrement stratégique des activités autour de Lom Pangar}

Dans le but de préserver les acquis et d'améliorer la croissance de ces différentes localités, la stratégie a consisté à montrer comment le potentiel de pêche qui peut être exploité ou capitalisé, peut booster le développement local. Cette stratégie consiste à contrôler la retenue (patrouilles dans l'eau et dans les îlots) et des voies d'accès (construction et création des postes de contrôle mixtes EDC/MINEPIA/Gendarmerie) ${ }^{30}$. Elle a également consisté à structurer et à organiser l'activité de la pêche à travers la création des coopératives. L'activité des coopératives permet de recenser, d'identifier les pêcheurs et de leur délivrer des permis de pêche et d'immatriculer les différentes embarcations.

${ }^{28}$ Rapport de l'atelier d'information et de mobilisation sociale autour des mesures environnementales et sociales du projet hydroélectrique de Lom Pangar organisé à Bertoua sous la coordination de la société Electricity Development Corporation du 28 février au 02 mars 2018.

${ }^{29}$ Ibid.

${ }^{30}$ Données issues de l'enquête sur le terrain à Ouami, novembre 2019. 
Cette stratégie a contribué à la création de nombreux débouchés dans ces localités : la pêche, le séchage, le stockage, le fumage, etc. Il s'agit entre autres de la commercialisation des produits de pêche (poissons frais et fumés, ...), maraichères/marnage (tomate, laitue, oignons, carottes, etc.), du développement des PME de transformation des produits agricoles (manioc, banane-plantain, ignames, maïs, ...), du développement des industries de transformation des produits forestiers (bois débités, produits forestiers non ligneux, papier, contreplaqués, ...). D'autres activités peuvent aussi être explorées notamment le développement des industries artisanales (fabrication des pirogues et autre matériels de pêche), le développement des PME dans le domaine de la restauration, le développement des industries extractives et la pratique des cultures de décrues dans la zone de la retenue.

La Mairie de Bélabo doit coordonner et superviser ces diverses activités qui demeurent clandestines, elle doit prélever des taxes à l'entrée du site d'Ouami avec une véritable traçabilité. En rendant cette activité légale, les revenus qui pourraient en découler permettraient de financer certains projets locaux, tels que la construction des infrastructures sanitaires, scolaires, hydrauliques, communautaires (marchés), voire administratives, etc., à partir des retombées financières de la pêche, du développement des industries et du commerce.

En définitive, la pêche et ces activités connexes représentent une véritable opportunité pour le développement économique et social de la Région de l'Est et principalement de l'arrondissement de Bélabo et des différentes localités autour de Lom Pangar. Electricity Development Company (EDC) en tant que maitre d'ouvrage, ainsi que certaines administrations impliquées comme le Ministère de l'élevage, des pêches et des industries animales, semble déjà jouer un rôle important. Il revient désormais aux collectivités locales décentralisées et aux forces vives, aux entrepreneurs de ces différentes localités et de la région de capitaliser le potentiel de pêche qui leur est offert par le projet, pour produire les richesses nécessaires au développement de leur localité.

\section{Adaptation des populations autochtones à la modernisation}

L'implémentation du barrage de Lom Pangar a produit des externalités positives qui se sont étendues à toutes les strates de la société, c'est-à-dire au niveau économique, environnemental et social. Les populations des localités environnant le site du barrage sont sujettes à une amélioration des conditions de vie et à un nouvel environnement. L'arrivée des peuples de divers horizons, leur confère une nouvelle conception de la cohabitation et nourrit en eux le sentiment d'appartenance à une même nation.

\section{Modernisation du cadre de vie des populations}

La réalisation du projet de construction du barrage réservoir de Lom Pangar a eu pour conséquence sociale la restructuration et l'amélioration du cadre de vie des populations dans les arrondissements de Belabo, de BétaréOya, de Ngoura, et des villages de Deng Deng, d'Ouami, etc. La route allant de Bertoua à Lom Pangar a été totalement terrassée sur le long pour permettre la circulation facile des véhicules, des biens et des personnes ${ }^{31}$. L'activité commerciale se développe grâce à l'aménagement des routes qui relient les différents villages au centre de la ville de Bertoua. Dans la localité de Bouli par exemple, des maisons individuelles, des latrines externes individuelles, des forages, des hangars, des salles de classe (écoles et lycée) ont été construites pendant la période de réalisation du projet et précisément au cours de l'année 2014-2015. On a relevé également des compensations collectives pour des villages et des campements qui ont été déplacés : puits d'eau, salles de classes, etc., des compensations pour les zones d'accueil : lieux sacrés, pertes de raphias, puits d'eau, salles de classes, etc., des actions spécifiques pour les déplacés et leurs zones d'accueil (personnes vulnérables ...).

En ce qui concerne l'approvisionnement en eau potable, on a pu observer la réalisation de 14 forages supplémentaires dans ces localités qui ont permis de réduire les difficultés d'accès à l'eau potable. Des programmes d'appui aux éleveurs, aux agriculteurs et aux pêcheurs ont été initiés et développés. L'approvisionnement en eau potable consiste en la mise en place des mini-réseaux dans certaines localités (Soa, Bangoua, Bana, Endom, Bikoka, Bidjoka, Magba, Nkwem, etc.) et les environs de la construction du Barrage. Le barrage de Lom Pangar a contribué également à l'amélioration de l'approvisionnement des populations urbaines de la ville de Bertoua, de Belabo, de Bétaré-Oya en eau potable ${ }^{32}$.

Dans le secteur de la santé, la réalisation du barrage de Lom Pangar a facilité la construction des centres de santé à l'instar de celui de Deng-Deng et la construction des blocs opératoires comme ceux de l'Hôpital de District de Bétaré-Oya (Projet d'aménagement hydroélectrique de Lom Pangar, «Etat de mise en œuvre des infrastructures du Plan de Gestion Environnementale et Sociales (PGES) » février 2018). Les populations ont également bénéficié de

\footnotetext{
${ }^{31}$ Constat fait en nous rendant au site du barrage quittant de Bertoua-Centre en novembre 2019.

${ }^{32} \mathrm{La}$ troisième conférence des Nations Unies sur le logement et le développement durable en milieu urbain (habitat III) dans son énoncé de position a pour objectif de réaffirmer le rôle primordial des villes dans le développement durable ; de financer en priorité et investir massivement dans les infrastructures collectives, vertes et sociales qui présentent le plus de retombées positives, octobre 2016.
} 
la fourniture des équipements biomédicaux. Toutes ces actions ont permis aux populations d'améliorer leurs conditions de vie et de santé.

\section{Le brassage ethnique au prisme du « vivre ensemble »}

Les localités ou les villages autour de l'ouvrage Lom Pangar sont désormais des sites d'attraction pour les populations nationales et étrangères. Les sites de la pêche et la biodiversité tropicale florissante sont des attractions qui sont actuellement exploitées par la population locale. La participation des populations à l'activité économique se traduit par la pratique de l'éco-tourisme qui permet d'éviter le chômage tout en revalorisant la culture locale. Les flux migratoires dans une zone entrainent souvent des divergences entre peuples car ils n'ont pas la même culture. Mais l'activité touristique dans ces localités permet la facilitation du contact avec autrui et l'appropriation d'une identité culturelle touristique. On assiste à un brassage et à une hétérogénéisation des différentes populations.

Le brassage des populations dans ces différentes localités, permet la mise en évidence de la notion du «vivreensemble » (Donzelot 2015), qui se développe depuis quelques années dans les propos du Chef de l'Etat et des élus politiques, depuis l'avènement des mouvements sociaux dont le Cameroun est victime. Ceux-ci mettent en avant deux sortes de pratiques: la production d'une plus grande mixité sociale et l'organisation plus méthodique de moments de partage des valeurs républicaines ; le Cameroun étant une nation qui prône le multiculturalisme.

Le « vivre-ensemble » ne se traduit pas simplement par la façon de penser, de manger, de se sentir et d'agir, il se traduit aussi par l'acceptation de l'autre. Ce qui démontre que les localités autour de Lom Pangar ne sont pas des jungles où chacun essaye de s'affirmer, mais des lieux ou des grands villages où l'acceptation de l'autre permet à chacun d'exister. Dès lors, « l'autre " n'est plus un ennemi au sens de Carl Schmitt (Schmitt 1992), mais un coéquipier, avec qui on peut échanger au sens de Max Weber. Les populations des territoires environnant du barrage, partagent désormais leur culture et s'adaptent à un nouveau mode de vie, tenant compte de leur diversité, mais aussi de leur environnement (Ngomou 2019 : 95).

De ce fait, la construction de l'infrastructure permet la création d'emplois, entraine la venue des chercheurs d'emploi et permet l'épanouissement des populations. Dans ces différentes localités autour de Lom Pangar, le chiffre de la population a augmente ${ }^{33}$. La venue d'une telle infrastructure dans cette localité permet de relever le niveau de vie des populations, participe à la lutte contre la pauvreté et contribue à la croissance économique de l'Etat.

\section{Conclusion}

En définitive, la réflexion sur le développement des territoires à partir de l'action publique de l'Etat a permis d'établir le lien qui peut exister entre une action pensée au niveau national et ses effets sur le plan local. La construction du Complexe Industrialo Portuaire de Kribi(CIPK) dans le domaine du transport maritime, et la construction du barrage réservoir ou entonnoir de Lom Pangar dans le domaine de l'énergie, se sont avérés être de véritables catalyseurs de développement sur le plan local. On assiste dans les localités de Kribi et de Lom Pangar où sont implantées ces différentes infrastructures de développement, à une dynamique territoriale traversée par des flux de croissance efficiente et de bien être. Les impacts d'ordre socio-économiques s'accumulent et concourent au développement des terres d'accueil et de leurs environs : pendant qu'à Kribi, on passe d'une ville touristique à une ville industrielle, dans les villages autour de Lom Pangar on observe le développement de la pêche artisanale continentale et des activités génératrices de revenus. Les populations autochtones et allogènes participent ellesmêmes au développement local, en saisissant ces opportunités qui s'offrent à elles.

$\mathrm{Au}$ regard des multiples atouts et richesses que regorgent le Cameroun, atouts qui se trouvent disséminés sur l'étendue du territoire, il serait primordial pour le pays de multiplier la construction des grands projets, pour permettre le développement territorial ou local de chaque région. Cette option aura pour avantage de favoriser le développement national à partir du développement local. C'est dire que chacun des projets implémentés dans un territoire, pris individuellement, participent au développement socio-économique de la localité. Le développement partitionné dans chaque petit ensemble concourt ainsi au rayonnement global du territoire national. Il revient donc aux autorités publiques d'impulser l'action par la construction des infrastructures à grande échelle pour faire croitre son PIB national et atteindre son émergence.

\section{Bibliographie}

Amanya Marguerite : Le redéploiement de l'Etat post-ajustement structurel au Cameroun, Thèse de Doctorat, Université de Yaoundé II Soa, 23 juin 2020 ;

\footnotetext{
${ }^{33}$ Entretien avec Roger KASSALA NDAN, chef de canton de Képéré Deng Deng le 20 Octobre 2019 à 16 h28.
} 
Amougou Gérard et Bobo Bobo René Faustin : Ambition développementaliste, état stationnaire et extraversion au Cameroun de Paul BIYA. Le projet de construction du port autonome de Kribi, Politique africaine, $\mathrm{n}^{\circ} 150$, Editions Karthala, n²/2018, pp. 29 -51 [En ligne];

Aschauer David: Public capital, productivity, and economic growth, Working Paper for the international Workshop on Infrastructure, Economic Growth and Regional development: The Case of Industrialized High Income Countries, Jonkoping, Sweden (10-12Juin), 1993;

Donzelot Jacques : développer "le vivre-ensemble" : qu'est-ce à dire ?, Tous urbains, 2015/2 (n¹0), p.12-13, DOI : 10.3917/tu.010.0012, consulté le 22.04.2019;

Ebele Onana Richard Désiré : Développement du sous-développement : contribution à l'étude des facteurs de production et de reproduction de la pauvreté au Cameroun de 1980 à 2008, Thèse de Doctorat en Science Politique, Université de Yaoundé II SOA, 13 juin 2015 ;

EDC : Atelier d'information et de mobilisation sociale autour des mesures environnementales et sociales du projet hydroélectrique de Lom Pangar, Bertoua, du 28 février au 02 mars 2018 ;

EDC/CIMA : Projet d'aménagement hydroélectrique de Lom Pangar, « Etat de mise en œuvre des infrastructures du Plan de Gestion Environnementale et Sociales (PGES), 28 février 2018;

Etoundi Emile : Cameroun : L'imposant port de Kribi est opérationnel !, Economie, le 25 avril 2018 ;

FAMBON Samuel et TACH Samuel : Conditions macroéconomiques et performances de l'investissement au Cameroun, in Bruno BEKOLO EBE (dir) : Mondialisation, Exclusion et développement Africain, Afrédit, Yaoundé, 2008 ;

Grawitz Madeleine et Leca Jean : Traité de science politique, tome I, La science politique, science sociale, L'ordre politique, PUF, Paris, 1985 ;

Halpern Charlotte (dir): L'instrumentation de l'action publique: controverses, résistances, effets, Presses de Sciences Po, Paris, 2014 ;

Henner Henri-François : L'impact des investissements d'infrastructure dans les économies en développement, in Gerardin Hubert et Brot Jean : Infrastructure et développement, l'Harmattan, Paris, 2001, pp. 39 - 56 ;

Investir au Cameroun : Kribi, futur pôle économique du Cameroun, $\mathrm{n}^{\circ} 42,2015$;

Kinhoun Epiphane et Ruppel Oliver (dir) : La question de la responsabilité sociale et environnementale de l'entreprise - perspective africaine, cas du Cameroun, 2018 ;

Krugman Paul: Increasing Returns and Economic Geography, Journal of political economy, n 99, 1991;

Kuate : Développement et économie, in David SIMO : La politique de développement à la croisée des chemins : le facteur culturel : colloque organisé à Yaoundé les 13 et 14 juin 1996, Editions CLE, Université de Californie, 1998 ;

Kuete Martin et Assongmo Théophile : Développement contre Environnement sous les Tropiques : l'exemple du littoral de la région de Kribi (Cameroun), Les Cahiers d'Outre-Mer [En ligne], 219 | Juillet-Septembre 2002, consulté le 29 avril 2019 ;

Lascoumes Pierre et Le Gales Patrick: Gouverner par les instruments, Presses de Sciences Po (P.F.N.S.P.) « Académique », 2004 ;

Magnan Marion : La production et la gestion de l'espace portuaire à vocation industrielle et logistique. Les grands ports maritimes français : gestionnaires d'espaces infrastructurels, Thèse de Doctorat en Géographie et Aménagement, Université Paris 1, 2016 ;

Merton Robert King : Eléments de théorie et de méthode sociologique, 1966, $1^{\text {re }}$ édition, 1944 ;

MINEPAT : Document de stratégie pour la croissance et l'emploi, Yaoundé, 2009 ;

MINEPAT : Etude d'Impact Environnemental et Social Sommaire du projet d'aménagement et d'électrification de la plage de Ngoye-kribi à des fins touristiques, Yaoundé, 2014;

MINEPIA : Rapport sur l'état de la pêche au Cameroun,Yaoundé, 2017 ;

Nga Ndongo Valentin: «Développement, émancipation et originalité », in David Simo: la politique de développement à la croisée des chemins : le facteur culturel : colloque organisé à Yaoundé les 13 et 14 juin 1996, Editions CLE, Université de Californie, 1998 ;

Ngomou Elat Merveille: Les implications socio-économiques et environnementales du complexe industrialoportuaire dans la ville de Kribi, Mémoire de fin d'études en Master II en Science politique, Faculté des Sciences Juridiques et Politiques, Université de Yaoundé II SOA, 8 Août 2019 ;

Nkutchet Modeste : l'Energie au Cameroun, l'Harmattan, Paris, 2004 ;

Pinson Gilles : Le projet urbain comme instrument d'action publique, in Lascoumes Pierre et Le Galès Patrick. : Gouverner par les instruments, Presses de Sciences Po (P.F.N.S.P.) «Académique », 2004, pp. 199-233;

Poirot Jacques : Du concept d'infrastructure aux services infrastructurels, in BROT Jean et GERARDIN Hubert, Infrastructure et développement, l'Harmattan, Paris, 2001, pp. 19 - 38 ;

Port Autonome de Kribi : Kribi Port. La porte s'ouvre sur une mer d'espérances, édition spéciale-numéro 4, Mars 2018 ;

Rist Gilbert : Le développement ; Histoire d'une croyance occidentale, Presses de Sciences Po (P.F.N.S.P.), $4^{\text {ème }}$ édition, 2013 ; 
Rizza Roberto : Néo-institutionnalisme sociologique et nouvelle sociologie économique : quelles relations?», Revue Interventions économiques [En ligne], 38 | 2008, mis en ligne le 01 décembre 2008, URL: http://journals.openedition.org/interventionseconomiques/292;

ROMER Paul: The origins of endogenous growth, Journal of Economic Perspectives, 8 (1), 1994, pp. 3-22.

Roux Christophe et Savarese Eric (dir) : Science politique, 2 édition, collection Paradigme, Bruylant, Bruxelles, 2018;

Sauret Jacques : L'action publique et sa modernisation : La réforme de l'État, mère de toutes les réformes, Terra Nova, 2013 ;

Tchinang Mesmer : L’énergie pour le développement au Cameroun, Editions Harmattan, Paris, 2011 ;

Touna Mama et Kamgnia-Dia : L'ajustement structurel en Afrique : l'investissement sacrifié, Revue Africaine des sciences économiques et de gestion, vol. $\mathrm{n}^{0} 2$ juillet-décembre 2000 ;

Touna Mama : l'Economie camerounaise, pour un nouveau départ, Afrédit, Yaoundé, janvier 2008.

Touna Mama : Pour la mort de l'Etat en Afrique, De quel Etat faut-il souhaiter la mort?, Mondialisation, exclusion et développement Africain, Stratégie des acteurs publics et privés, Afrédit, 2006. 